\title{
Pairing symmetry and spin-polarized quasiparticle transport in high- temperature superconducting cuprates
}

Nai-Chang Yeh, Chu-Cheng Fu, Ching-Tzu Chen, Z. Huang, Richard P. Vasquez, et al.

Nai-Chang Yeh, Chu-Cheng Fu, Ching-Tzu Chen, Z. Huang, Richard P. Vasquez, Setsuko Tajima, "Pairing symmetry and spin-polarized quasiparticle transport in high-temperature superconducting cuprates," Proc. SPIE 4058, Superconducting and Related Oxides: Physics and Nanoengineering IV, (6 September 2000); doi: 10.1117/12.397825 


\title{
Pairing Symmetry and Spin-Polarized Quasiparticle Transport in High-Temperature Superconducting Cuprates
}

\author{
N.-C. Yeh ${ }^{a}$, C.-C. Fu', C.-T. Chen ${ }^{a}$, Z. Huang ${ }^{a}$, R. P. Vasquez ${ }^{b}$, and S. Tajima ${ }^{c}$ \\ ${ }^{a}$ Department of Physics, California Institute of Technology, Pasadena, CA 91125, USA \\ bJet Propulsion Laboratory, California Institute of Technology, Pasadena, CA 91109, USA \\ 'Superconductivity Research Laboratory, International Superconductivity Technology Center, \\ Tokyo 135-0062, Japan
}

\begin{abstract}
The pairing symmetry and the superconducting gap in high-temperature superconducting cuprates are investigated as a function of the hole doping level $(x)$ and temperature (T), using directional scanning tunneling spectroscopy (STS). It is found that the predominant pairing symmetry is $d_{x^{2}-y^{2}}$, which is insensitive to the variations in $T$ and $x$. In contrast, the maximum superconducting gap $\left(\Delta_{d}\right)$ in $\mathrm{YBa}_{2} \mathrm{Cu}_{3} \mathrm{O}_{7-\delta}$ and $\mathrm{La}_{2-x} \mathrm{Sr}_{x} \mathrm{CuO}_{4-8}$ scales with the superconducting transition temperature $\left(T_{c}\right)$, and the ratio of $\left(2 \Delta_{d} k_{B} T_{c}\right)$ increases with decreasing doping level. The dominance of $d_{x} 2_{-y} 2$ pairing is consistent with strong spatial variations in the local quasiparticle spectra near non-magnetic impurities such as $\mathrm{Zn}$ and $\mathrm{Mg}$ in a $(\mathrm{Zn}, \mathrm{Mg})$-doped $\mathrm{YBa}_{2} \mathrm{Cu}_{3} \mathrm{O}_{7-8}$ single crystal. To further elucidate the nature of the pairing state, the c-axis spin-polarized quasiparticle transport in the superconducting state of $\mathrm{YBa}_{2} \mathrm{Cu}_{3} \mathrm{O}_{7-\delta}$ is investigated by studying the critical currents and STS under the injection of electrical currents from the underlying ferromagnetic $\mathrm{La}_{0.7} \mathrm{Sr}_{0.3} \mathrm{MnO}_{3}$ layer in various ferromagnetinsulator-superconductor (F-I-S) heterostructures. The temperature dependent spin diffusion length $\left(\delta_{s}\right)$ and signatures of nonequilibrium quasiparticle distribution under spin injection in $d$-wave superconductors are determined for the first time.
\end{abstract}

Keywords: pairing symmetry, scanning tunneling spectroscopy (STS), cuprate superconductors, zero-bias conductance peak (ZBCP), spin-polarized quasiparticles, F-I-S heterostructures, spin diffusion length, nonequilibrium superconductivity.

\section{INTRODUCTION}

\subsection{Pairing Symmetry in Cuprate Superconductors}

The purity of pairing symmetry in cuprate superconductors has been an issue of intense investigation and heated debate in recent years ${ }^{1-19}$. Despite overwhelming experimental evidence supporting the $d_{x^{2}-y^{2}}$ symmetry as the predominant pairing component ${ }^{1-8}$, whether there may exist a secondary pairing component, particularly one that results in the breaking of timereversal symmetry ${ }^{11-15}$, remains controversial. Theoretically, pairing mechanism based on the antiferromagnetic spin fluctuations would require a $d_{x^{2}-y^{2}}$ pairing symmetry ${ }^{20}$, whereas anion superconductivity would favor the $\left(d_{x^{2}-y^{2}} 2+i d_{x y}\right)$ pairing symmetry ${ }^{21-23}$, yielding time-reversal symmetry breaking. It has also been conjectured that the dominant $d_{x^{2}-y^{2}}$ pairing channel may be suppressed within approximately a coherence length of a surface that permits the formation of Andreev bound states, yielding a local time-reversal symmetry breaking and $(d+i s)$ pairing symmetry even in the absence of any magnetic field ${ }^{11,12}$. This "surface-induced time reversal-symmetry breaking" effect would result in the splitting of the zerobias conductance peak (ZBCP) as the result of Doppler shift of quasiparticle energies, and the magnitude of such splitting would increase linearly with increasing magnetic field ${ }^{12}$. However, the lack of universal experimental evidence for the timereversal symmetry breaking phenomena in both zero and finite magnetic fields in a vast quantity of experimental data ${ }^{1-8,15}$ casts concerns on the scenario of surface-induced time reversal symmetry breaking. Recent theoretical investigation ${ }^{16}$ of competing superconducting pairing channels based on a lattice model with a pairing kernal involving onsite repulsion and nearest-neighbor attraction on a square lattice has suggested that the $d$-wave pairing channel generally prevails if the single particle density of states is close to or more than half-filling ${ }^{16}$. Furthermore, a number of theoretical calculations $s^{17,18}$ have demonstrated that surface impurities can significantly influence the local quasiparticle spectra of cuprate superconductors. A recent comprehensive calculation ${ }^{19}$ for the ground state of doped antiferromagnetic insulators suggests that while $d_{x^{2}-y^{2}}$ is the pairing symmetry for the ground state, the $\left(d_{x^{2}-y^{2}} 2+i d_{x y}\right)$ pairing may be a short-lived meta-stable state that can occur through 
either thermal excitations or existence of impurities. All these unsettled issues obvious require further experimental and theoretical investigation.

\subsection{Spin and Charge Channels in the Normal and Superconducting States of Cuprates}

Another central issue of debates is the physical origin of a "pseudogap" and the non-Fermi liquid behavior in the normal state of underdoped and optimally doped cuprate superconductor $\mathrm{s}^{24-26}$. That is, there exists an energy scale comparable to a characteristic temperature $T^{*}$, below which the density of states in the normal state is suppressed, indicating the opening of a pseudogap. The pseudogap temperature $T^{*}$ increases with decreasing doping level $(x)$ and is nearly independent of the temperature $(T)$. Further, experimental evidence suggests that $T^{*}$ is related to the occurrence of a spin gap in the cuprates, and $T^{*}$ is significantly above the superconducting transition $T_{c}$ for the underdoped cuprates. The general consensus is that the non-Fermi liquid behavior of the cuprates for temperatures between $T_{c}$ and $T^{*}$ is the result of different behavior in the spin and charge channels, known as the spin-charge separation ${ }^{27-31}$. In addition, the absence of long-range phase coherence ${ }^{32,33}$ in these "doped Mott insulators" between $T_{c}$ and $T^{*}$ is believed to contribute to the unconventional normal state properties. This view also leads to the proposed dynamic and Josephson-coupled "stripe phases" in the cuprates that may be responsible for the existence of a quasi one-dimensional Luttinger liquid, the latter is known to result in spin-charge separation phenomena in lower-dimensional physical systems.

To further elucidate the issue of spin-charge separation below $T^{*}$, it is important to design experiments that can directly assess the characteristic lengths and times for the spin and charge channels ${ }^{34-41}$. Our approach to this investigation is to perform spin injection experiments on perovskite ferromagnet-insulator-superconductor (F-I-S) thin-film heterostructures ${ }^{34}$ 36. By characterizing how pair-breaking effects due to spin-polarized quasiparticles depend on the temperature and the thickness of the superconducting layer, we are able to deduce the spin diffusion length $\delta_{5}(T)$ along the $c-a x i s$ of $\mathrm{YBa}_{2} \mathrm{Cu}_{3} \mathrm{O}_{7-\delta}$ in its superconducting state. In addition, by considering the pair-breaking efficiency and transmission of spin-polarized quasiparticles in $\mathrm{YBa}_{2} \mathrm{Cu}_{3} \mathrm{O}_{7-\delta}$, we are able to demonstrate signatures of nonequilibrium superconductivity in $d$-wave superconductors for the first time.

\section{QUASIPARTICLE TUNNELING SPECTRA OF CUPRATE SUPERCONDUCTORS}

\subsection{Sample Characterizations and Surface Preparations for STS Studies}

The samples used for our STS studies include optimally doped and underdoped pure $\mathrm{YBa}_{2} \mathrm{Cu}_{3} \mathrm{O}_{7-\delta}$ single crystals with superconducting transition temperatures of $91 \pm 1 \mathrm{~K}$ and $60 \pm 3 \mathrm{~K}$, one nearly optimally doped $\mathrm{YBa}_{2} \mathrm{Cu}_{3} \mathrm{O}_{7-\delta}$ single crystal with controlled $0.26 \% \mathrm{Zn}$ and $0.4 \% \mathrm{Mg}$ impurities, and underdoped a-axis oriented $\mathrm{La}_{2-x} \mathrm{Sr}_{\mathrm{x}} \mathrm{CuO}_{4-\delta}$ films with $x=0.15$, 0.125 , and 0.10 . The samples for the STS studies have been characterized by $\mathrm{x}$-ray refraction (XRD). The crystalline axes of the single crystals selected for the tunneling studies are determined by optical microscopy, and those of the oriented films are based on the XRD information. The technique used in this investigation involves a low-temperature scanning tunneling microscope (LT-STM) for studying the directional tunneling spectroscopy of cuprate superconductors along various crystalline axes. The tunneling tip is made of $\operatorname{Pt}(85 \%)-\operatorname{Ir}(15 \%)$, and the highest voltage resolution at the lowest temperature is $200 \mu V$. The temperature range covered in this work is between $2.4 \mathrm{~K}$ and $10 \mathrm{~K}$.

The surface preparation is very important for STS and STM studies, because STM is a surface sensitive probe with a depth no more than $10 \mathrm{~nm}$. Consequently, it is necessary to reduce any appreciable non-stoichiometric surface layers in order to obtain reasonable information for the generic properties of the materials to be investigated. For the single crystal samples used in our experiments, they were first cut to reveal the desirable crystalline plane, polished to optical flatness, and then annealed at proper temperatures under a gas flow of different oxygen partial pressure to yield the necessary doping level. The sample was subsequently chemically etched with $1 \% \mathrm{Br}_{2}$ in pure ethanol (or $0.5 \% \mathrm{Br}_{2}$ in pure ethanol for thin film samples), and then thoroughly rinsed in pure ethanol. This process has been demonstrated by XPS studies to yield optimal electronic properties at the surface of various cuprate superconductors ${ }^{42,43}$, and to passivate the surface to prevent degradation in air over an extended period of time ( $\sim$ tens of minutes). Hence, the sample could be properly transferred to the STM cryostat to achieve high vacuum and low temperature condition to ensure preservation of the surface quality before experiments.

\subsection{Optimally Doped and Underdoped $\mathrm{YBa}_{2} \mathrm{Cu}_{3} \mathrm{O}_{7-\delta}$ Single Crystals}

Following our previous investigation, we employ the generalized BTK theory ${ }^{44}$ by $\mathrm{Hu}^{9}$, Kashiwaya and Tanaka ${ }^{10}$ to analyze the directional tunneling spectra. The relevant physical quantities thus derived include the percentage of possible secondary 
pairing components, the maximum gap $\Delta_{d}$, lifetime broadening parameter $\Gamma$, and the tunneling impedance $Z$. More explicitly, we consider the tunneling current $\left(I_{N S}\right)$ from a normal metal to a superconductor can be described in terms of the generalized BTK theory as a function of the bias voltage $(V)$ using the following formula ${ }^{3,9,10}$ :

$$
I_{N S}=G_{N N} \iint \exp \left[-\left(k_{t} / \beta\right)^{2}\right] d^{2} k_{t} \times \int d E_{k}\left[1+A\left(E_{k} \Delta_{b} Z\right)-B\left(E_{k} \Delta_{k} Z\right)\right]\left[f\left(E_{k}-e V\right)-f\left(E_{k}\right)\right] .
$$

Here $G_{N N}$ is proportional to the normal-state junction conductance, $A$ and $B$ are the Andreev-reflection and normal reflection probabilities ${ }^{9,10}, f$ is the Fermi function for quasiparticle distributions, $E_{k}$ is the quasiparticle energy, and $\beta$ is the tunneling cone width that measures the effective spread in the transverse quasiparticle momentum $\left(k_{t}\right)$ and the surface roughness ${ }^{3}$. To estimate the possible contribution from different pairing components, we assume that the pairing potential is given by $\Delta_{k}=\Delta_{d}$ $\cos \left(2 \theta_{k}\right)+i \Delta_{s}$ for $(d+i s), \Delta_{k}=\Delta_{d} \cos \left(2 \theta_{k}\right)+i \Delta^{\prime} \sin \left(2 \theta_{k}\right)$ for $(d+i d)$, and $\Delta_{k}=\Delta_{d} \cos \left(2 \theta_{k}\right) \pm \Delta_{s}$ for $(d \pm s)$.

The fitting parameters for different junctions of both optimally doped and underdoped $\mathrm{YBa}_{2} \mathrm{Cu}_{3} \mathrm{O}_{7-\delta}$ single crystals are summarized in Table 1. In particular, we note that the zero-bias conductance peak (ZBCP) for the $\{110\}$ junctions taken at temperatures between $2.4 \mathrm{~K}$ and $4.2 \mathrm{~K}$ do not reveal any splitting down to a voltage resolution of $\sim 0.2 \mathrm{meV}$, as exemplified by a representative set of data in Figure 1. Within our experimental resolution, the absence of any splitting in the ZBCP for many tens of $\{110\}$ tunneling spectra investigated in our studies places an upper bound $\sim 5 \%$ for the existence of any timereversal symmetry-breaking component. Furthermore, we note that the maximum conductance of the ZBCP in our work is nearly one order of magnitude larger than the background normal conductance. This is in sharp contrast to the typical ZBCP signals reported in planar junctions, where the maximum conductance is generally between $0.1 \%$ to $1 \%$ of the background normal conductance ${ }^{11,15}$. This contrast is likely due to the large differences in the junction areas, where the area of a typical planar junction is about $10^{-8} \sim 10^{-10} \mathrm{~m}^{2}$, and that of an STM tunneling junction is $10^{-18} \sim 10^{-16} \mathrm{~m}^{2}$. In addition, the differences in the tunnel barrier for the STS studies, which is a vacuum gap, versus those for planar junctions, which typically consist of polymers and/or oxide layers ${ }^{11,13,15}$, may have important effects on the resulting quasiparticle spectra.

Our results on optimally doped and underdoped $\mathrm{YBa}_{2} \mathrm{Cu}_{3} \mathrm{O}_{7-\delta}$ single crystals are consistent with a number of experiments from other research groups on various forms of $\mathrm{YBa}_{2} \mathrm{Cu}_{3} \mathrm{O}_{7-\delta}$, including experiments performed directly on the cuprate superconductors using techniques such as STM on $\{110\}$ oriented films for temperature down to $80 \mathrm{mK}$ and in a field up to 7 Tesla ${ }^{8}$, grain-boundary junctions for temperature down to $0.1 \mathrm{~K}$ and in a field up to $12 \mathrm{Tesla}^{7}$, and scanning SQUID microscopy on tri-crystals of $\mathrm{YBa}_{2} \mathrm{Cu}_{3} \mathrm{O}_{7-\delta}$ films in zero field from $0.5 \mathrm{~K}$ up to $90 \mathrm{~K}^{6}$. None of these studies revealed any evidence of spontaneous time-reversal symmetry breaking, with an upper bound for a secondary pairing components lowered to $0.1 \%$, according to the STS studies of $\{110\}$ oriented $\mathrm{YBa}_{2} \mathrm{Cu}_{3} \mathrm{O}_{7-\delta}$ films. In addition, recent point contact measurements using $\mathrm{InO}_{2}$ on $\mathrm{YBa}_{2} \mathrm{Cu}_{3} \mathrm{O}_{7-\delta}$ oriented films also find that there is no $\mathrm{ZBCP}$ splitting in the zero magnetic field in either optimally doped or underdoped limit ${ }^{15}$. Only $\mathrm{YBa}_{2}\left(\mathrm{Ca}_{\mathrm{x}} \mathrm{Cu}_{3-\mathrm{x}}\right) \mathrm{O}_{7-\delta}$ oriented films that are overdoped cuprates exhibit finite ZBCP splitting in zero magnetic field ${ }^{15}$. Furthermore, the magnetic field dependence of the ZBCP splitting appears to be very different between the optimally doped and overdoped samples ${ }^{15}$. These results obviously call for reevaluation of the relevance of Doppler shift to the time-reversal symmetry breaking.

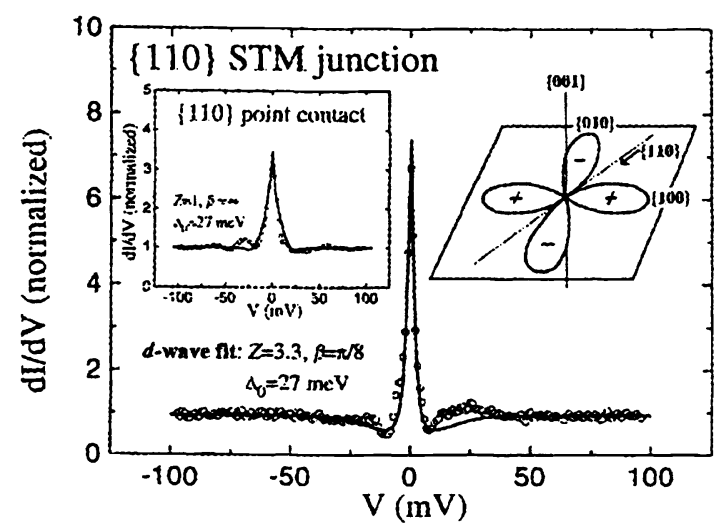

Figure 1 Normalized quasiparticle tunneling spectra of a nearly optimally doped $\mathrm{YBa}_{2} \mathrm{Cu}_{3} \mathrm{O}_{7-\delta}$ single crystal at $4.2 \mathrm{~K}$, showing the $\mathrm{ZBCP}$ for the $\{110\}$ junction in the tunneling limit (main panel), and that in the point contact limit (left inset). The magnitude of the maximum ZBCP is nearly one order of magnitude larger than the background normal conductance, which is about two to three orders of magnitude larger than typically ZBCP signals in planar junctions. The absence of any splitting down to $2.4 \mathrm{~K}$ and with a voltage resolution $\sim 0.2 \mathrm{meV}$ places $a<5 \%$ upper bound for any time-reversal symmetry breaking component. 
In addition to the consideration of pairing symmetry, it is also interesting to compare the c-axis tunneling spectra of both optimally doped and underdoped samples. As illustrated in Figure 2, the averaged $d$-wave gap as indicated by the position of the coherence peaks at $\pm \Delta_{d}$ decreases with the decreasing $T_{c}$. In addition, we note that similar satellite features exist in both samples, and the energy scale of each feature appears to scale with the energy gap. We note that observation of satellite features have also been reported for the c-axis quasiparticle tunneling spectra of $\mathrm{Bi}_{2} \mathrm{Sr}_{2} \mathrm{CaCu}_{2} \mathrm{O}_{8+x}$ single crystals ${ }^{45-47}$, and the "dip" and "hump" features at energies $\Omega_{\text {dip }}$ and $\Omega_{\text {nump }}$ have been attributed to many-body effects of quasiparticle interaction with the background spin excitations ${ }^{47,48}$. We shall come back to this issue later in subsection 2.4 .

Table 1 Summary of the fitting results for the $\mathrm{YBa}_{2} \mathrm{Cu}_{3} \mathrm{O}_{7-\delta}$ and $\mathrm{La}_{2-x} \mathrm{Sr}_{x} \mathrm{CuO}_{4-\delta}$ samples using the generalized BTK theory, with $\Delta_{d}$ being the maximum $d_{x 2-y_{2}}$ gap, $Z$ the tunneling barrier parameter, $\Gamma$ the lifetime broadening parameter, and $p$ the hole concentration per $\mathrm{CuO}_{2}$. The errors are given to account for all spectral variations, instrumental resolutions, and numerical fitting uncertainties.

\begin{tabular}{lcccccc}
\hline Junctions & $\underline{\Delta}_{d}(\mathrm{meV})$ & $\underline{\left(2 \Delta_{d} \underline{k}_{B} \underline{\underline{C}}_{\underline{\alpha}}\right)}$ & $\underline{Z}$ & $\underline{\Gamma(\mathrm{meV})}$ & $\underline{p}$ & $\underline{\text { Secondary Pairing Component }}$ \\
$90 \mathrm{~K}-\mathrm{YBCO}\{100\}$ & $29 \pm 3$ & $7.5 \pm 0.8$ & $5.2 \pm 0.5$ & 0 & 0.15 & $<5 \%$ \\
$90 \mathrm{~K}-\mathrm{YBCO}\{100\}$ & $28 \pm 3$ & $7.2 \pm 0.8$ & $0.4 \pm 0.1$ & 0 & 0.15 & $<5 \%$ \\
$90 \mathrm{~K}-\mathrm{YBCO}\{110\}$ & $27 \pm 4$ & $7.2 \pm 0.8$ & $2.7 \pm 0.1$ & 0 & 0.15 & $<5 \%$ \\
$90 \mathrm{~K}-\mathrm{YBCO}\{001\}$ & $19 \pm 4$ & $4.9 \pm 1.0$ & $4.5 \pm 0.2$ & 0 & 0.15 & \\
$60 \mathrm{~K}-\mathrm{YBCO}\{110\}$ & $22 \pm 5$ & $8.5 \pm 1.9$ & $3.6 \pm 0.2$ & $1.9 \pm 0.2$ & 0.10 & $<9 \%$ \\
$6 \mathrm{~K}-\mathrm{YBCO}\{001\}$ & $17 \pm 1$ & $6.6 \pm 0.3$ & $4.0 \pm 0.5$ & $1.0 \pm 0.5$ & 0.10 & \\
$(\mathrm{Zn}, \mathrm{Mg})-\mathrm{YBCO}\{001\}$ & $25 \pm 3$ & $6.7 \pm 0.8$ & $5.0 \pm 0.5$ & $1.0 \pm 0.5$ & 0.18 & \\
$28 \mathrm{~K}-\mathrm{LSCO}\{110\}$ & $15 \pm 4$ & $12.4 \pm 3.3$ & $1.1 \pm 0.2$ & $5.0 \pm 0.6$ & 0.10 & $<10 \%$ \\
$17 \mathrm{~K}-\mathrm{LSCO}\{110\}$ & $15 \pm 4$ & $20.5 \pm 5.5$ & $1.2 \pm 0.2$ & $4.1 \pm 0.5$ & 0.08 & $<10 \%$ \\
$10 \mathrm{~K}-\mathrm{LSCO}\{100\}$ & $13 \pm 2$ & $30.1 \pm 4.6$ & $1.5 \pm 0.5$ & $2.5 \pm 0.6$ & 0.06 & \\
\hline$=$
\end{tabular}

\subsection{Optimally Doped and Underdoped $\mathrm{La}_{2-x} \mathrm{Sr}_{x} \mathrm{CuO}_{4-\delta}$ Oriented Films}

We have also investigated a different family of high-temperature superconducting cuprates, the a-axis oriented $\mathrm{La}_{2-x} \mathrm{Sr}_{x} \mathrm{CuO}_{4-\delta}$ films, in order to evaluate the purity of $d$-wave pairing symmetry in these samples that exhibit a larger electronic anisotropy than that in $\mathrm{YBa}_{2} \mathrm{Cu}_{3} \mathrm{O}_{7-\delta}$ and consist of one $\mathrm{CuO}_{2}$ layer per unit cell. The samples include optimally doped and underdoped

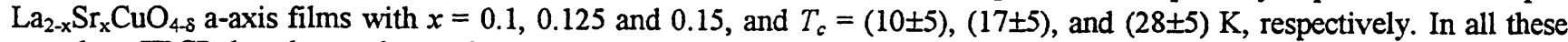
samples, $\mathrm{ZBCP}$ has been. observed, and none of these spectra exhibit any visible splitting within our experimental resolution ${ }^{4,5}$. Due to the larger transition width and more significant disorder in these oriented films, we place an upper bound for any secondary pairing component in $\mathrm{La}_{2 \cdot x} \mathrm{Sr}_{x} \mathrm{CuO}_{4-\delta}$ at $\sim 10 \%$.

The above analysis suggests that among all samples of our investigation, there is no discernible evidence of mixed pairing components, regardless of the temperature, doping level, and even the degree of disorder. The fact that only experiments involving some planar junctions (with dissimilar materials placed on top of the cuprate superconductor) have revealed signs of mixed pairing components ${ }^{11,13,15}$ suggests that more cautious reevaluation of the status of experimental evidence for the surface-induced time-reversal symmetry breaking will be necessary. Furthermore, a number of theoretical calculations ${ }^{17,18}$ have suggested that surface impurities can significantly influence the local quasiparticle spectra of cuprate superconductors. In particular, it is found that the quasiparticle spectra of $d$-wave superconductors are far more sensitive to non-magnetic impurities than those of conventional $s$-wave superconductors ${ }^{17,18}$. Given the fact that planar junctions generally cover a large junction area and involve dissimilar materials as the tunneling barrier, it is difficult to entirely rule out the role of impurities in the observed splitting of ZBCP. In particular, recent theoretical analysis on doped antiferromagnets has found that while the pure $d_{x^{2}-y^{2}}$-pairing is the ground state, a time-reversal symmetry breaking state $\left(d_{x^{2}-y^{2}} 2+i d_{x y}\right)$ may exist as a short-lived metastable state ${ }^{19}$, the latter could be thermally excited or stabilized by the presence of impurities. It seems that mounting theoretical studies have led to the suggestion that the time-reversal symmetry-breaking state, if exists in the cuprate superconductors, is more likely to be in the form of $\left(d_{x^{2}-y^{2}} 2+i d_{x y}\right)$ pairing symmetry rather than $\left(d_{x^{2}-y^{2}}+i s\right)$. However, this issue remains controversial and will require in-depth experimental and theoretical investigation. 
Another interesting aspect that is worth noting in our studies is that the ratio of $\left(2 \Delta_{d} k_{B} T_{c}\right)$ for both $\mathrm{La}_{2-\mathrm{x}} \mathrm{Sr}_{\mathrm{x}} \mathrm{CuO}_{4-\delta}$ and $\mathrm{YBa}_{2} \mathrm{Cu}_{3} \mathrm{O}_{7-\delta}$ increases rapidly with decreasing doping, and the ratio well exceeds the mean-field value $(\sim 4.3)$ for $d$-wave superconductors, as listed in Table 1 . These results obtained in the superconducting state appear to be consistent with the unconventional normal state properties of underdoped cuprates, suggesting that the underdoped regime is consistent with a strongly interacting many-body system.

\subsection{Comparison of C-Axis Quasiparticle Tunneling Spectra of $\mathrm{YBa}_{2} \mathrm{Cu}_{3} \mathrm{O}_{7-\delta}$ and $\mathrm{Bi}_{2} \mathrm{Sr}_{2} \mathrm{CaCu}_{2} \mathrm{O}_{8+x}$ Single Crystals}

As shown in Table 1, our tunneling studies indicate that in optimally doped and underdoped $\mathrm{YBa}_{2} \mathrm{Cu}_{3} \mathrm{O}_{7-8}$ and a-axis oriented $\mathrm{La}_{2-\mathrm{x}} \mathrm{Sr}_{\mathrm{x}} \mathrm{CuO}_{4-\delta}$ films, the maximum $d$-wave gap $\left(\Delta_{d}\right)$ appears to scale with $T_{c}$. This finding is in contrast to the $c$-axis tunneling and ARPES spectra of $\mathrm{Bi}_{2} \mathrm{Sr}_{2} \mathrm{CaCu}_{2} \mathrm{O}_{8+x}$ single crystals, which exhibit increasing gap values $\left(\Delta^{*}\right)$ with decreasing doping ${ }^{45-47}$. In addition, the satellite features in both optimally doped and underdoped $\mathrm{YBa}_{2} \mathrm{Cu}_{3} \mathrm{O}_{7-\delta}$ appear to scale with $\Delta_{d}$, as shown in Figure 2; whereas those in $\mathrm{Bi}_{2} \mathrm{Sr}_{2} \mathrm{CaCu}_{2} \mathrm{O}_{8+x}$ scale with $\Delta^{*}{ }^{47,48}$. We note that the gap features at $V= \pm(\Delta * / e)$ in $\mathrm{Bi}_{2} \mathrm{Sr}_{2} \mathrm{CaCu}_{2} \mathrm{O}_{8+x}$ have been determined by various techniques, including STS ${ }^{46}$, and SNN and SIS point contact measurements ${ }^{47}$, and ARPES ${ }^{45,48}$. It is found that these gap features at $V= \pm\left(\Delta^{*} / e\right)$ do not close at $T_{c}$ from STS studies ${ }^{46}$, resembling a pseudogap and in contrast to those in $\mathrm{YBa}_{2} \mathrm{Cu}_{3} \mathrm{O}_{7-\delta}$.

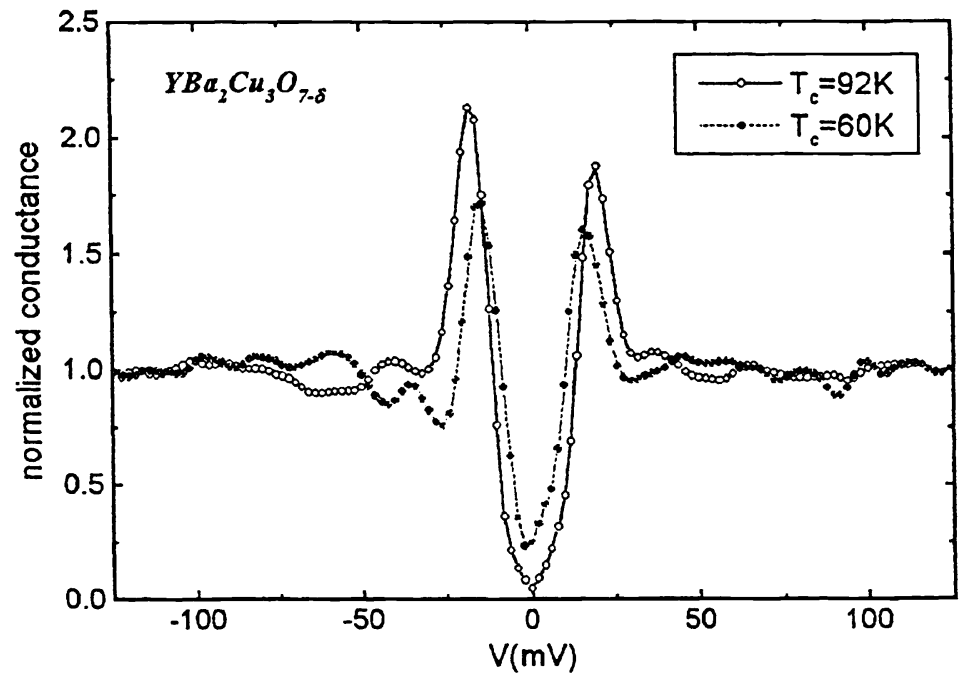

Figure 2 Comparison of the normalized c-axis quasiparticle tunneling spectra of nearly optimally doped and underdoped $\mathrm{YBa}_{2} \mathrm{Cu}_{3} \mathrm{O}_{7-\delta}$ single crystals at $4.2 \mathrm{~K}$. Note that the satellite features scale in energy with the superconducting coherence peaks at $V= \pm\left(\Delta_{d} / e\right)$.

On the other hand, if we compare the satellite features at $\pm\left(\Omega_{\text {dip }}\right)$ and $\pm\left(\Omega_{\text {hump }}\right)$ relative to the c-axis tunneling gap of $\Delta_{d}\left(\Delta^{*}\right)$ in $\mathrm{YBa}_{2} \mathrm{Cu}_{3} \mathrm{O}_{7-\delta}\left(\mathrm{Bi}_{2} \mathrm{Sr}_{2} \mathrm{CaCu}_{2} \mathrm{O}_{8+x}\right)$, we find that these features scale almost linearly with their corresponding gaps, as shown in Figure 3(a). It remains an open issue why the c-axis gaps obtained from tunneling measurements have different doping dependence in $\mathrm{YBa}_{2} \mathrm{Cu}_{3} \mathrm{O}_{7-5}$ and in $\mathrm{Bi}_{2} \mathrm{Sr}_{2} \mathrm{CaCu}_{2} \mathrm{O}_{8+x}$, as shown in Figure 3(b) where the gap versus hole concentration $(p)$ per unit $\mathrm{CuO}_{2}$ exhibit very different behavior. We note that the data for the overdoped YBCO system is taken from a $(\mathrm{Zn}, \mathrm{Mg})-$ doped $\mathrm{YBa}_{2} \mathrm{Cu}_{3} \mathrm{O}_{7-\delta}$ single crystal described below.

\section{4. ( $\mathrm{Zn}, \mathrm{Mg}$ )-Doped $\mathrm{YBa}_{2} \mathrm{Cu}_{3} \mathrm{O}_{7-\delta}$ Single Crystal - Investigation of the Effects of Non-Magnetic Impurities}

It is well known that non-magnetic impurities in s-wave superconductors do not incur any effect on the quasiparticle spectra, in contrast to the significant pair-breaking effects of magnetic impurities ${ }^{49-51}$. On the other hand, recent theoretical calculations have shown that strong pair breaking effects of non-magnetic impurities can exist in pure $d$-wave superconductors ${ }^{17,18}$. The effects of both magnetic and non-magnetic impurities on the quasiparticle spectra of d-wave superconductors have been confirmed by recent experiments on $\mathrm{Ni}$ and $\mathrm{Zn}$-doped $\mathrm{Bi}_{2} \mathrm{Sr}_{2} \mathrm{CaCu}_{2} \mathrm{O}_{8+x}$ single crystals $52-58$. In particular, spatially resolved imaging and spectroscopy taken with a high-resolution scanning tunneling microscopy ${ }^{57}$ reveal 
that $\mathrm{Zn}$ impurities are strong scattering centers that completely suppress the superconducting coherence peaks, and the scattering energy at the $\mathrm{Zn}$ site is $\Omega \sim-1.5 \mathrm{meV}$. In contrast, $\mathrm{Ni}$ impurities yield excess resonance scattering peaks in both the hole-like and electron-like branches of the quasiparticle spectra without significantly perturbing the superconducting coherence peaks that are characteristics of pure $\mathrm{Bi}_{2} \mathrm{Sr}_{2} \mathrm{CaCu}_{2} \mathrm{O}_{8+\mathrm{x}}$. Furthermore, the quasiparticle spectra around $\mathrm{Ni}$ impurities exhibit oscillatory variations ${ }^{58}$. Such behavior has not been considered by existing theories for impurity scattering in $d$-wave superconductors. We note that the oscillatory quasiparticle distribution is in fact analogous to the well-known Friedel oscillation $^{59}$ of the carrier distribution near a charged impurity in a metal. Therefore a complete theoretical analysis should reveal such a phenomenon. The finding that $\mathrm{Zn}$-impurities appear to be stronger pair-breakers than Ni-impurities is consistent with neutron scattering and optimal measurements on the bulk properties of similar systems ${ }^{52-56}$. It is interesting to note that the single scattering peak at the $\mathrm{Zn}$ site ${ }^{57}$ is consistent with pure $d_{x^{2}-y^{2}}$ pairing ${ }^{18}$, which is in contrast to the prediction of two scattering peaks at energies $\pm|\Omega|$ for $\left(d_{x^{2}-y^{2}}+i d_{x y}\right)$ or $\left(d_{x^{2}-y^{2}}+i s\right)$ pairing, according to the T-matrix theoretical calculations ${ }^{18}$.
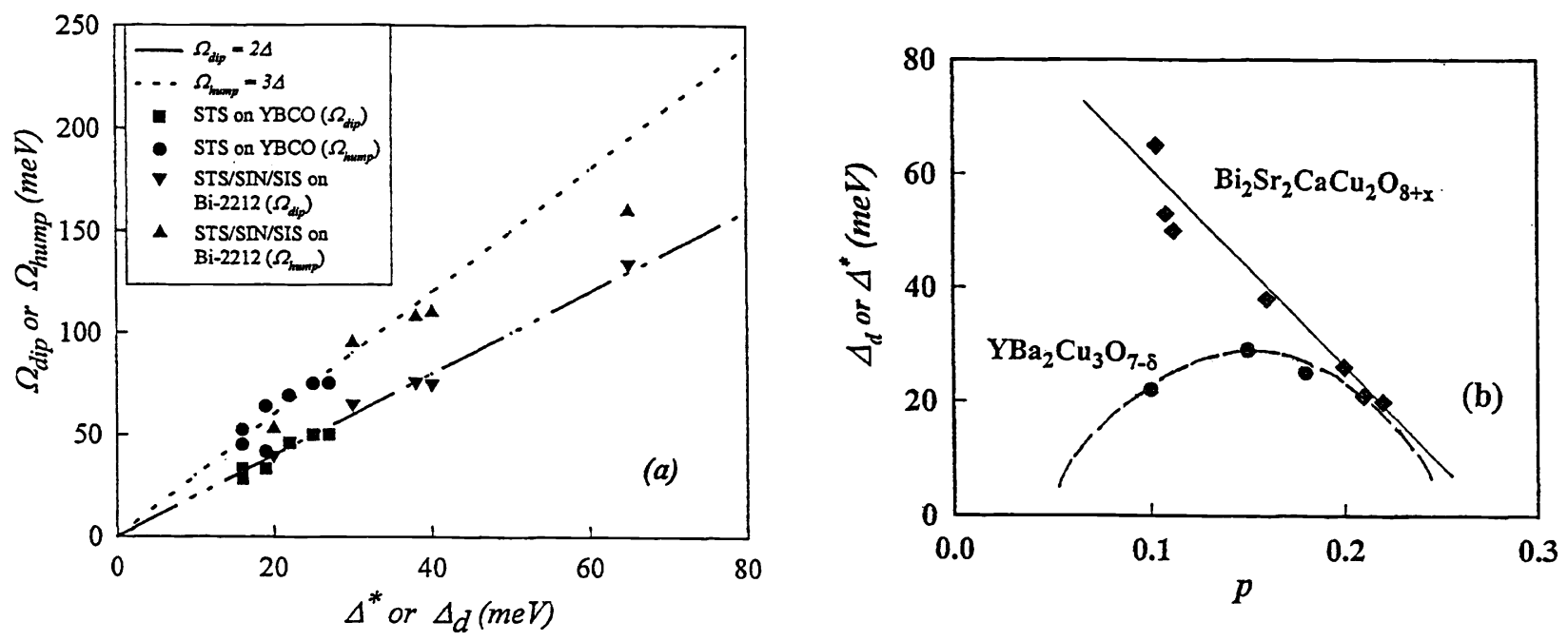

Figure 3 Comparison of the satellite features obtained from the c-axis quasiparticle tunneling spectra of $\mathrm{YBa}_{2} \mathrm{Cu}_{3} \mathrm{O}_{7-\delta}$ single crystals and those of $\mathrm{Bi}_{2} \mathrm{Sr}_{2} \mathrm{CaCu}_{2} \mathrm{O}_{8+x}$ at $4.2 \mathrm{~K}$. Note that the satellite features scale in energy with $\Delta_{d}$ for $\mathrm{YBa}_{2} \mathrm{Cu}_{3} \mathrm{O}_{7-\delta}$ and with $\Delta *$ for $\mathrm{Bi}_{2} \mathrm{Sr}_{2} \mathrm{CaCu}_{2} \mathrm{O}_{8+\mathbf{x}}$. The data for $\mathrm{Bi}_{2} \mathrm{Sr}_{2} \mathrm{CaCu}_{2} \mathrm{O}_{8+\mathrm{x}}$ are taken from Refs. 46 and 47.

On the other hand, the puzzling contrast between the c-axis tunneling spectra of $\mathrm{YBa}_{2} \mathrm{Cu}_{3} \mathrm{O}_{7-\delta}$ and $\mathrm{Bi}_{2} \mathrm{Sr}_{2} \mathrm{CaCu}_{2} \mathrm{O}_{8+\mathrm{x}}$ single crystals suggests that the quasiparticle spectra near impurities in $\mathrm{YBa}_{2} \mathrm{Cu}_{3} \mathrm{O}_{7-8}$ need not be the same as those in $\mathrm{Bi}_{2} \mathrm{Sr}_{2} \mathrm{CaCu}_{2} \mathrm{O}_{8+x}$. In addition, studies of spatially resolved quasiparticle spectra have only focused on the effects of nonmagnetic impurities that do not contribute to doping. It is therefore informative if similar spectroscopy studies can be conducted near those impurities that affect the doping level of the cuprates.

To investigate these issues, we have conducted preliminary tunneling spectra on $\mathrm{Zn}$-doped $\mathrm{YBa}_{2} \mathrm{Cu}_{3} \mathrm{O}_{7-\delta}$ single crystal (with $0.26 \% \mathrm{Zn}$ and $0.4 \% \mathrm{Mg}$ ). The c-axis tunneling spectroscopy on the ab-plane of the single crystal at $4.2 \mathrm{~K}$ reveals reproducible spatially varying quasiparticle spectra, depending on the position of the STM tip relative to either $\mathrm{Zn}$ or $\mathrm{Mg}$ impurities. When the STM tip is sufficiently far away from impurities, the quasiparticle spectrum is consistent with the standard c-axis tunneling spectra of d-wave superconductors, as indicated by Curve " $\mathrm{O}$ " in Figure 4. The spectrum corresponds to an average gap value of $(24 \pm 2) \mathrm{meV}$, which is consistent with our previous studies on pure $\mathrm{YBa}_{2} \mathrm{Cu}_{3} \mathrm{O}_{7-8}$ single crystal. As the STM tip approaches an impurity site, we observe two typical types of spectral evolution, corresponding to two types of scattering centers, although the nature of each is yet to be identified. The "type- $A$ " scattering center is associated with a sharp resonance scattering at an energy of $\sim+2 \mathrm{meV}$, and the spatial evolution of the spectra as the tip approaches the impurity center $A$ can be illustrated by considering the spectra in the order of Curves $\mathrm{O}, \mathrm{A}-1, \mathrm{~A}-2$, and A-3. We note that with the initial increase in the intensity of the impurity resonance scattering peak at $\sim+2 \mathrm{meV}$, the superconducting coherence peak in the electron branch $(\sim+24 \mathrm{meV})$ first diminishes noticeably, and then both the electronand hole-branch coherence peaks vanish as the impurity resonance becomes dominant. This suggests that type- $A$ scattering center is a strong pair-breaker, similar to the finding in the $\mathrm{Zn}$-doped $\mathrm{Bi}_{2} \mathrm{Sr}_{2} \mathrm{CaCu}_{2} \mathrm{O}_{8+\mathrm{x}}$. We also note that occasionally 
resonant scattering peaks at the impurities sites have also been observed at energies of $\sim-2 \mathrm{meV}$ for A-type and $\sim-10 \mathrm{meV}$ for B-type impurities. The observation of only one c-axis resonant scattering peak at each non-magnetic impurity site is again consistent with theoretical predictions for pure $d_{x^{2}-y^{2}}$ pairing ${ }^{18}$.

Similarly spatial evolution of the quasiparticle spectra is also observed for the other type of scattering center $B$, as manifested by Curves O, B-1, B-2 and B-3. The only difference in these two types of spectra is the energy of the impurity resonance. One corresponds to a repulsive scattering potential $\Omega_{A} \sim 2 \mathrm{meV}$, the other is associated with a higher repulsive scattering potential $\Omega_{B} \sim 10 \mathrm{meV}$. Judging from the frequency of occurrence, we tentatively assign the $A$-site to $\mathrm{Zn}$ and the $B$-site to $\mathrm{Mg}$. We note that the scattering potential for $\mathrm{Zn}$-doped $\mathrm{Bi}_{2} \mathrm{Sr}_{2} \mathrm{CaCu}_{2} \mathrm{O}_{8+\mathrm{x}}$ has been reported to be primarily negative, with $\Omega \sim$ $(-1.5 \pm 0.5) \mathrm{meV} .{ }^{57}$ More investigation appears necessary to understand the differences between the systems of $\mathrm{Zn}$-doped $\mathrm{YBa}_{2} \mathrm{Cu}_{3} \mathrm{O}_{7-\delta}$ and $\mathrm{Zn}$-doped $\mathrm{Bi}_{2} \mathrm{Sr}_{2} \mathrm{CaCu}_{2} \mathrm{O}_{8+\mathrm{x}}$.

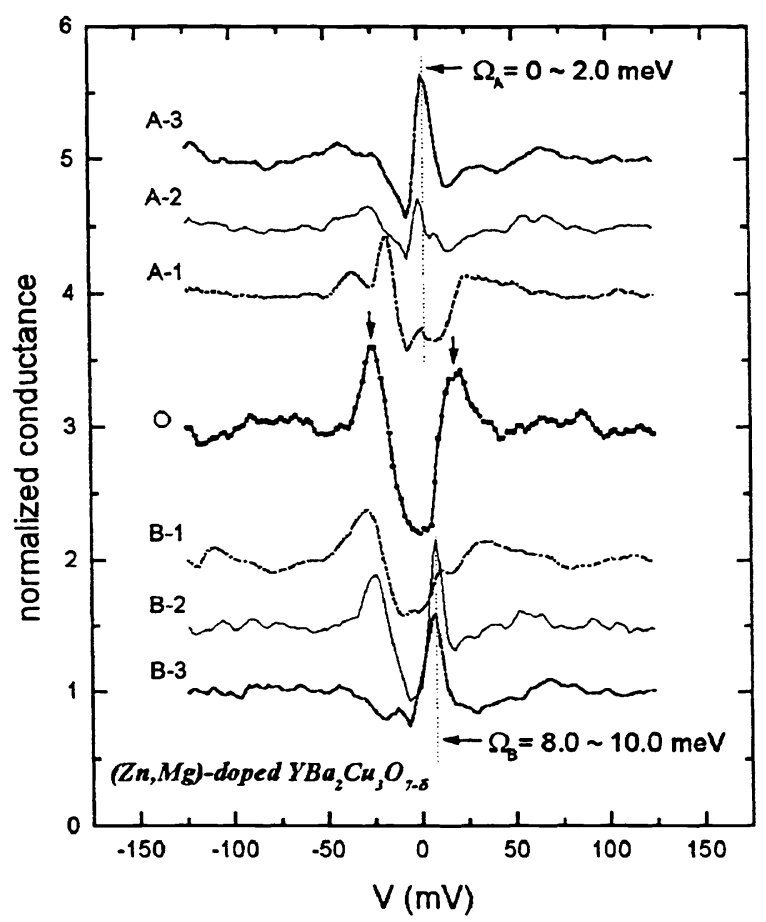

Figure 4 Quasiparticle tunneling spectra of a $(\mathrm{Zn}, \mathrm{Mg})$-doped $\mathrm{YBa}_{2} \mathrm{Cu}_{3} \mathrm{O}_{7-\delta}$ single crystal at $4.2 \mathrm{~K}$, showing the spatial evolution of the normalized differential conductance versus bias voltage $(V)$. Curve $\mathrm{O}$ : typical spectrum away from impurities. Curves A-1 to A-3 illustrate the spatial evolution of the spectra upon approaching scattering center $A$, while Curves $\mathrm{B}-1$ to $\mathrm{B}-3$ illustrate the spatial evolution upon approaching scattering center $B$.

\section{NONEQUILIBRIUM SUPERCONDUCTIVITY AND C-AXIS SPIN-POLARIZED QUASIPARTICLE TRANSPORT IN D-WAVE SUPERCONDUCTORS}

As stated in the introduction, the spin and charge relaxation processes of quasiparticles in the cuprates are of fundamental importance, because of their relevance to the underlying pairing mechanism and the possibility of spin-charge separation in high-temperature superconductors. A novel approach to derive such information is to study the nonequilibrium superconductivity ${ }^{60}$ in these cuprates by injecting either simple or spin-polarized quasiparticles. The injection of spinpolarized quasiparticles is of particular interest, because the resulting effects may be compared with those of static magnetic impurities that incur time-reversal symmetry-breaking in superconductors ${ }^{49-51}$. On the other hand, there has been limited theoretical understanding at the microscopic level for the spin-polarized quasiparticle transport in superconductors. The only 
theoretical studies have emphasized on either the transmission of spin-polarized quasiparticles across the ferromagnet/superconductor interface ${ }^{61-63}$, or possible experimental consequences under excess spin diffusion along the $\{100\}$ and $\{110\}$ direction of a d-wave superconductor ${ }^{39-41}$. The lack of microscopic understanding is largely due to the complications of combined magnetic pair-breaking ${ }^{49-51}$ and nonequilibrium effects ${ }^{60}$ induced by spin-polarized quasiparticle currents $^{64-66}$. In this section, we briefly review the fundamentals of nonequilibrium superconductivity and magnetic pairing breaking effects of static magnetic impurities, and describe latest experimental status of spin injection studies in cuprate superconductors. We then present new results that provide direct evidence for nonequilibrium quasiparticle distribution due to quasiparticle injection, as well as direct experimental determination of spin diffusion length $\delta_{s}$. The physical significance of these results will be assessed.

\subsection{Magnetic Pair Breaking and Nonequilibrium Superconductivity in Superconductors}

The presence of microscopic magnetic fields in a superconductor is known to degrade superconductivity due to the breaking of time-reversal symmetry of the Cooper pairs ${ }^{49-51}$. Traditionally, magnetic pair-breaking effects were studied by doping the superconductor with paramagnetic impurities that act as local magnetic moments ${ }^{50,51}$. The subsequent experimental development of spin-polarized electron tunneling from a ferromagnetic layer through a insulating barrier into a superconductor ${ }^{64,65}$ has inspired a new direction of research involving the dynamic process of spin-injection in superconductors and in magnetic materials ${ }^{66}$. Despite interesting experimental observation in the past two decades, not much theoretical understanding of the physics of spin-polarized quasiparticle transport in superconductors has been developed at the microscopic level, except reasonable understanding of the transport properties at the ferromagnet/superconductor interface $^{61-63}$. The lack of microscopic understanding is largely due to the complications of combined magnetic pair-breaking and nonequilibrium effects induced by spin-polarized quasiparticle currents in a superconductor. Recent theoretical work based on a mean-field, quasi-static description of excess spin-polarized quasiparticles in $s$-wave superconductors has predicted interesting spatial variations in the superconducting order parameter and the formation of solitons with net spins ${ }^{67}$. However, such quasi-static description does not address either the dynamic nature of the excess quasiparticles or the phase variation in the order parameter under the presence of a finite quasiparticle current. In the case of cuprate superconductors, the effect of spin-polarized quasiparticle currents on superconductivity may be further complicated by the intrinsic spatial variations in the superconducting order parameter due to the predominant $d$-wave pairing symmetry.

Many novel phenomena associated with nonequilibrium superconductivity have been investigated extensively since the $1970^{\prime} s^{60}$. Nonetheless, these studies have primarily focused on the effects of simple (spin-degenerate) quasiparticle injection and extraction in conventional $s$-wave superconductors. In the case of simple quasiparticles, both enhancement and suppression of superconductivity have been observed, and the phenomena can be generally described in terms of a nonequilibrium energy and an excess quasiparticle charge density $\left(Q^{*}\right)^{60}$. In the case of injection of spin-polarized quasiparticles, it has been demonstrated that electrical currents can become spin polarized after passing through ferromagnets ${ }^{64,65}$, and the spin-polarized current with sufficient injection energy may tunnel through an insulating barrier into a conventional superconductor, giving rise to suppression of superconductivity ${ }^{60}$. Recently, various research group $\mathrm{s}^{34-38}$ have extended investigation of the effects of spin-injection to perovskite ferromagnet-insulator-superconductor (F-I-S) heterostructures. As discussed in our recent publication ${ }^{34-36}$, earlier reports of monotonic suppression in $J_{c}$ with increasing dc injection currents ${ }^{37,38}$ appear to have been influenced by the effects of Joule heating. Hence, genuine effects associated with spin-polarized quasiparticles may have been obscured. We have overcome this problem by using a pulsed current technique for studies of macroscopic and microscopic physical properties of perovskite F-I-S heterostructures, and have demonstrated the effect of dynamic pair-breaking of spin-polarized quasiparticles ${ }^{34,36}$.

\subsection{Review of Experimental Status}

New phenomena manifesting nonequilibrium superconductivity and pair breaking induced by spin-polarized quasiparticle currents in perovskite F-I-S heterostructures have been reported in our recent work ${ }^{34-36}$. Using a pulsed current technique for measurements of the critical current density $J_{c}$, we are able to minimize Joule heating effects to less than $10 \mathrm{mK}$ for the maximum injection current $\left(I_{m} \sim 300 \mathrm{~mA}\right)$ from the ferromagnetic layer to the superconductor, and have been able to reveal several novel phenomena. For instance, a monotonic decrease in $J_{c}$ with decreasing insulator thickness is observed; and for FI-S with sufficiently thin insulating barriers, a slight increase in $J_{c}$ is observed under small injection current from the ferromagnet, followed by a strong suppression of $J_{c}$ under large injection current. In contrast, no effect of injection on $J_{c}$ can be detected in either the N-I-S control sample or F-I-S samples with thick insulating barrier. These phenomena are consistent with the pair breaking effects induced by spin-polarized quasiparticles. 
In addition to the studies of $J_{\sigma}$ we have also investigated the c-axis quasiparticle tunneling spectroscopy of perovskite F-I-S heterostructures comprising $\mathrm{YBa}_{2} \mathrm{Cu}_{3} \mathrm{O}_{7-8}$ and $\mathrm{La}_{0.7} \mathrm{Ca}_{03} \mathrm{MnO}_{3}$ at $4.2 \mathrm{~K}{ }^{35,36}$. The spectra were consistent with the $d$-wave pairing symmetry of $\mathrm{YBa}_{2} \mathrm{Cu}_{3} \mathrm{O}_{7-\delta}$, with a gap maximum $\Delta_{0}=22 \mathrm{meV}$, under an injection current up to at least $35 \mathrm{~mA}\left(7 \times 10^{3}\right.$ $\left.\mathrm{A} / \mathrm{cm}^{2}\right)^{35,36}$. Spectral smearing observed at higher injection currents could be fitted to an elevated effective quasiparticle temperature $(\sim 60 \mathrm{~K})$, even though negligible sample heating was detected with our in-situ thermometry ${ }^{35,36}$. The overall spectral evolution with the injection current appears to be non-thermal in character, showing a non-monotonic change in both the zero-bias tunneling conductance and the area under the conductance spectrum. In contrast, no detectable effects of injection were observed in the N-I-S sample ${ }^{36}$.

\subsection{New Results from Transport Studies}

Although we have demonstrated both macroscopic and microscopic evidence for dynamic pair breaking by the injection of spin-polarized quasiparticles into cuprate superconductors, more quantitative information associated with the nonequilibrium nature of the system still needs to be established. For instance, it is important to determine the magnitude and temperature dependence of the spin diffusion length and spin relaxation time. In addition, experimental determination of the nonequilibrium quasiparticle distributions under spin injection will be helpful in reaching better understanding for the quasiparticle excitations in cuprate superconductors. Finally, the microscopic mechanism for pair breaking induced by the spin-injection is still not well understood. One possibility may be related to the paramagnetic effect ${ }^{6}$ of the excess magnetization induced by the injection of spin-polarized quasiparticles. It is therefore necessary to consider the relevance of paramagnetic effect to the pair-breaking mechanism.

We have attempted to address these issues by empirically investigating the temperature evolution of pair-breaking effects in F-I-S heterostructures of a range of thickness in the superconducting layer. The samples used in this investigation include three samples of different thickness for the superconducting layer, and of identical thickness for the insulating and ferromagnetic layers. The chemical compositions for the heterostructures are $\mathrm{F}: \mathrm{La}_{0.7} \mathrm{Sr}_{0.3} \mathrm{MnO}_{3}$ (LSMO), I: $\mathrm{SrTiO}_{3}$ (STO), and $\mathrm{S}: \mathrm{YBa}_{2} \mathrm{Cu}_{3} \mathrm{O}_{7-\delta}$, and the thickness for the $\mathrm{YBCO}$ layers are $50 \mathrm{~nm}, 100 \mathrm{~nm}$, and $200 \mathrm{~nm}$.

To illustrate the physical concept for our data analysis, we first define relevant physical quantities that have been investigated in this work. Consider the inset in Figure 5, where the current applied to the ferromagnet is $I_{m}$, and the total current injected into the superconductor is denoted as $I_{i n j}$. The transmission of spin-polarized quasiparticles across the F-I-S junction is therefore defined as $T \equiv I_{i n j} / I_{m}$. The injected current $\left(I_{i n j}\right)$ can be determined empirically from the current-voltage $(I-V)$ characteristics of the superconductor, as shown in the main panel of Figure 5, where an $I-V$ curve taken at a constant temperature becomes offset in the presence of $I_{i n j}$. In addition to the offset, the initial critical current $I_{c}^{0}(T)$ becomes suppressed by the injected spin-polarized quasiparticle current, yielding a new critical current $I_{c}\left(T, I_{i n j}\right)$ that depends on both $T$ and $I_{i n j}$. Hence, we define the "pair-breaking efficiency" $(\eta)$ of spin-injection as

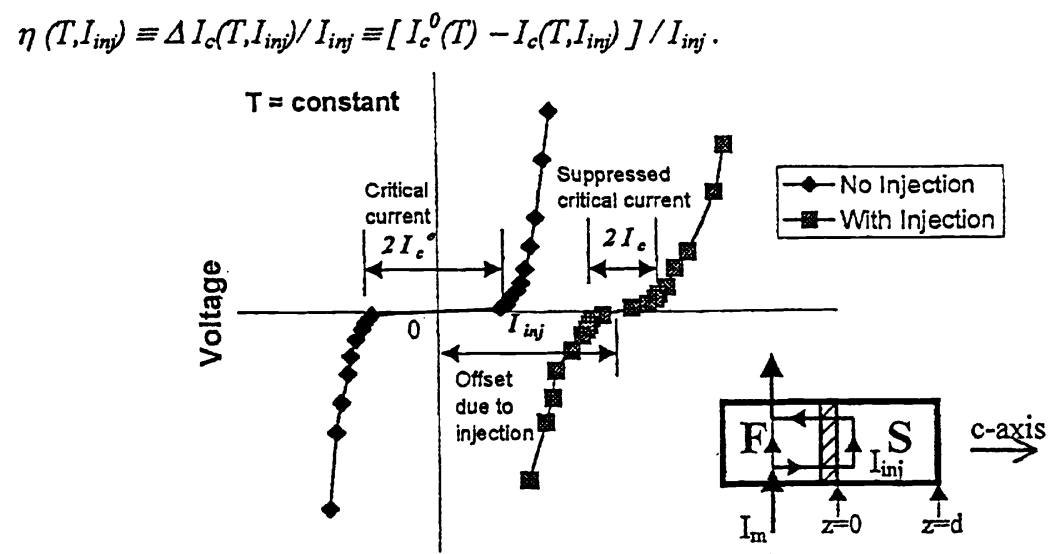

\section{Current}

Figure 5 Main panel: examples of the current-voltage characteristics of $\mathrm{YBa}_{2} \mathrm{Cu}_{3} \mathrm{O}_{7-\delta}$ in the F-I-S heterostructure at a constant temperature. The critical current in the absence of injection current is denoted as $I_{c}{ }^{0}$, whereas that under a finite injection current $I_{i n}$ is denoted as $I_{c}$. Inset: schematic illustration of the side view of the F-I-S heterostructures, showing the applied current $I_{m}$ through the ferromagnet and the actual current injected $I_{i n j}$ into the superconductor. The superconductor thickness is $d$, and the injection direction for currents is along $z$. 
Next, we consider the relationship between the excess magnetic moments $m$ in the superconductor as the result of $I_{i n g}$. If we define the longitudinal spin relaxation time as $T_{l}$, and the diffusion constant as $D_{s}$, then the following diffusion equation and boundary conditions for $m\left(T, z, I_{i n j}\right)$ in the steady state are satisfied:

$$
D_{s} \partial^{2} m / \partial z^{2}=m / T_{1} ; \quad D_{s} \partial m /\left.\partial z\right|_{z=0}=I_{i n j}\left(\mu_{B} / e\right) ; \quad D_{s} \partial m /\left.\partial z\right|_{z=d}=0 .
$$

Here $\mu_{B}$ denotes the Bohr magneton. Assuming complete spin polarization for the injected quasiparticles, the spatial dependence for $m(z)$ and the spatial average $\langle m\rangle$ can be given by the following expressions:

$$
m(z)=\left(T_{1} / \delta_{s}\right) I_{i n j}\left(\mu_{B} / e\right) \cosh \left[(d-z) / \delta_{s}\right] / \sinh \left(d / \delta_{s}\right) ;\langle m\rangle=\left(T_{1} / d\right) I_{i n j}\left(\mu_{B} / e\right) ; \quad \delta_{s}=\left(D_{s} T_{1}\right)^{1 / 2} .
$$

It is reasonable to assume that the pair-breaking efficiency $\eta$ is proportional to $\langle m\rangle$. Hence, we expect that $\eta$ increases with increasing spin relaxation time $T_{1}$ and the total injected spin-polarized quasiparticle current $I_{i m \text {, }}$ and $\eta$ decreases with increasing thickness of the superconducting layer $d$. However, this consideration only takes into account of the quasi-static effect of excess magnetic moments. To fully incorporate the nonequilibrium effects under spin-injection, we consider the nonequilibrium quasiparticle distribution function $f_{k}\left(T, I_{i n j}\right)$, which is different from the equilibrium distribution function $f_{k 0}$ (T). That is,

$$
f_{k}\left(T, I_{i n j}\right)=1 /\left\{1+\exp \left[\left(E_{k}-\mu^{*}\right) /\left(k_{B} T\right)\right]\right\}, \quad f_{k 0}(T)=1 /\left\{1+\exp \left[E_{k} /\left(k_{B} T\right)\right]\right\},
$$

where $\mu^{*}=\mu^{*}\left(I_{i n j}\right)$ is the chemical potential shift as the result of injected quasiparticles, and $E_{k}$ is the quasiparticle energy in thermodynamic equilibrium, where $E_{k}^{2}=\xi_{k}^{2}+\Delta_{k}^{2}$, and $\xi_{k}$ is the normal-state single-electron energy measured relative to the Fermi level. Knowing the average magnetic moment $\langle m\rangle$ and the nonequilibrium quasiparticle distribution $f_{k}\left(T, I_{i n j}\right)$, the pairbreaking efficiency $\eta$ can be expressed in terms of $\langle m\rangle$ and $f_{k}$ as follows:

$$
\eta \sim\langle m\rangle \Sigma_{k}\left(1-2 f_{k}\right),
$$

where $\Sigma_{k}$ indicates the sum over all relevant $k$ values in the momentum space. The chemical potential shift $\mu *$ is expected to increase linearly with increasing $I_{i n j}$ for small injection currents, and then gradually saturates for large $I_{i n j}$ before the critical current in the superconductor is completely suppressed to zero. Consequently, from Eqs. (2), (4), (5), and (6), we find that the pair-breaking efficiency $\eta$ increases monotonically with increasing $I_{i n j}$ in the high-temperature limit, whereas $\eta$ drops precipitously with initial increase in $I_{i n j}$ at low temperatures. As shown in Figure 6, the empirical results for $\eta$-vs. $I_{i n j}$ taken at various constant temperatures for a heterostructure YBCO/STO/LSMO (with thickness of individual layers given by $50 \mathrm{~nm} / 2$ $\mathrm{nm} / 100 \mathrm{~nm}$ ) are indeed consistent with this consideration.

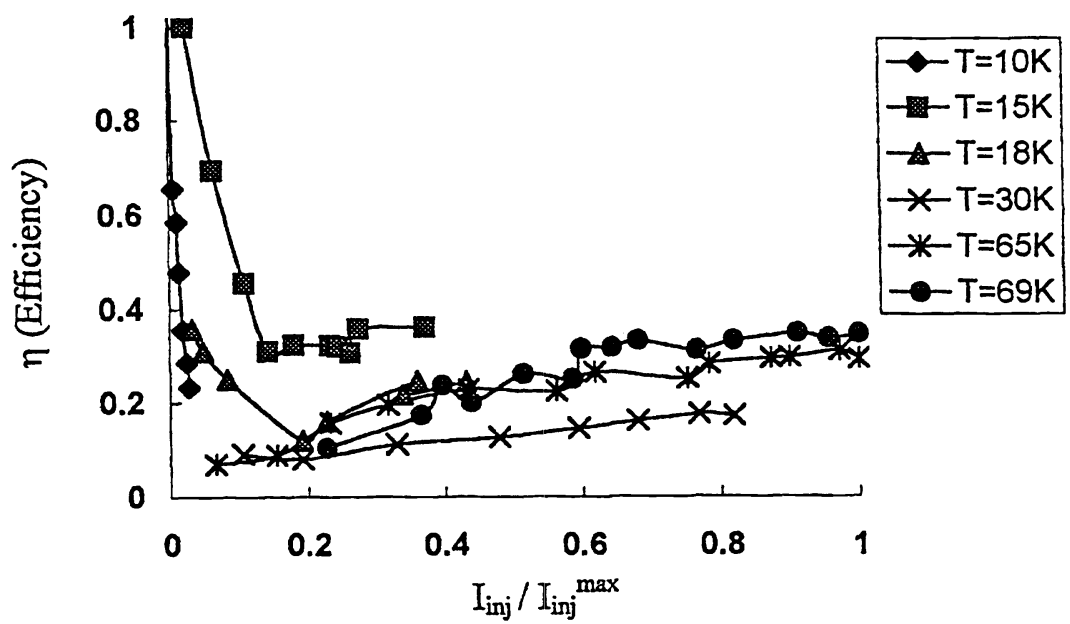

Figure 6 The pair-breaking efficiency $\eta$ as a function of the injected spin-polarized quasiparticle current $I_{i n j}$ taken at various temperatures for a YBCO/STO/LSMO $(50 \mathrm{~nm} / 2 \mathrm{~nm} / 100 \mathrm{~nm})$ heterostructure. Note that for $\left(T / T_{c}\right) \ll 1, \eta$ decreases rapidly with the initial increase in $I_{i n j}$. In contrast, $\eta$ increases monotonically $I_{i n j}$ for $\left(T / T_{c}\right) \rightarrow 1$.

Knowing the magnitude $\langle m\rangle$ for given experimental conditions, we can estimate the effective magnetic induction $B_{\text {eff }}$ in the superconductor. That is,

$$
B_{\text {eff }}=\mu_{0}\langle m\rangle / \Omega \quad\left(\Omega \text {. sample volume, } \mu_{0}\right. \text { : vacuum permeability) }
$$


Using Eq.(7), we find that $B_{\text {eff }}<\sim 0.01$ Tesla for all our experimental conditions. Consequently, we conclude that it is unlikely for the pair breaking effect of spin-polarized quasiparticles to be primarily induced by the paramagnetic effect, because paramagnetic effect in is only expected to be significant if $B_{\text {eff }}$ becomes a significant fraction of the upper critical field ${ }^{68}$.

Finally, we consider the effect of temperature-dependent spin diffusion length $\delta_{s}(T)$ on the pair-breaking phenomena as observed in the transport measurements. As shown in Figures $7(\mathrm{a})$ and $7(\mathrm{~b})$ for two F-I-S samples of $\mathrm{YBa}_{2} \mathrm{Cu}_{3} \mathrm{O}_{7-\delta}$ thickness $50 \mathrm{~nm}$ and $200 \mathrm{~nm}$, respectively, suppression of critical currents due to spin-polarized quasiparticles for the thinner superconducting sample is already very significant at low temperatures, whereas that for the thicker sample only becomes substatial at temperatures very close to $T_{c}$. Assuming that the spin diffusion length becomes comparable to the thickness of the sample when the critical current $I_{c}(T)$ is suppressed by $90 \%$ under a given injection current, we can estimate $\delta_{s}(T)$ by studying F-I-S heterostructures with a range of thickness in the superconductor, as shown in Figure 8. We note that $\delta_{5}(T)$ increases rapidly near $T_{\odot}$ consistent with rapid decreasing condensate and increasing lifetime of spin-polarized quasiparticles. However, the divergence is much more rapidly than the mean-field prediction, which may be related to the unconventional nature of the cuprate superconductor. More accurate temperature dependence of $\delta_{s}(T)$ awaits studies of additional F-I-S samples with different thicknesses in the superconducting layer.
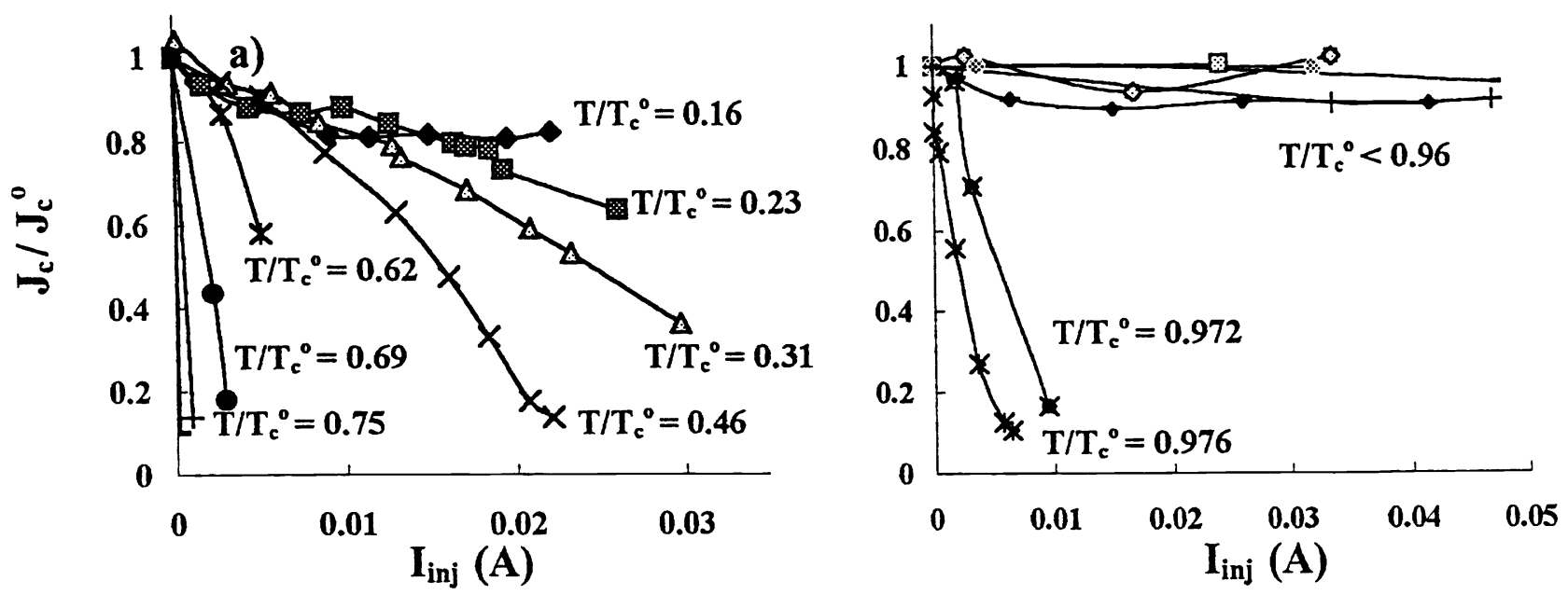

Figure 7 Normalized critical current $\left(I_{c} I_{c 0}\right)$ vs. injection current $\left(I_{i n}\right)$ in two YBCO/STO/LSMO heterostructures: (a) sample thickness: 50 $\mathrm{nm} / 2 \mathrm{~nm} / 100 \mathrm{~nm}$; (b) sample thickness: $200 \mathrm{~nm} / 2 \mathrm{~nm} / 100 \mathrm{~nm}$. Note that significant suppression of critical current in (a) already occurs at relatively low temperatures, whereas no suppression in critical current takes place in (b) until $\left(T / T_{c}\right) \rightarrow 1$.

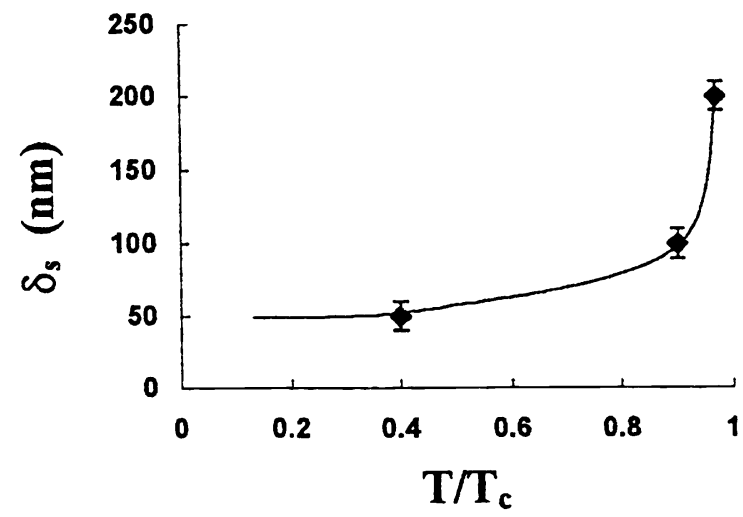

Figure 8 Estimated spin diffusion length $\left(\delta_{s}\right)$ as a function of the reduced temperature $\left(T / T_{c}\right)$ from this work. The error bars indicate the uncertainty in the YBCO thickness. Note the strong divergence of $\delta_{s}$ as $\left(T / T_{c}\right) \rightarrow 1$. 


\section{SUMMARY}

Directional quasiparticle tunneling spectra on cuprate superconductors have been investigated as a function of the doping level $(x)$ and temperature $(T)$, using a low-temperature scanning tunneling microscope. The samples studies include optimally doped and underdoped $\mathrm{YBa}_{2} \mathrm{Cu}_{3} \mathrm{O}_{7-\delta}$ single crystals, $(\mathrm{Zn}, \mathrm{Mg})$-substituted $\mathrm{YBa}_{2} \mathrm{Cu}_{3} \mathrm{O}_{7-\delta}$ single crystals with excess holes due to the presence of $\mathrm{Mg}$, as well as optimally doped and underdoped a-axis oriented $\mathrm{La}_{2-\mathrm{x}} \mathrm{Sr}_{x} \mathrm{CuO}_{4-\delta}$ films with $x=0.15,0.125$, and 0.10 . We find that the pairing symmetry for all samples investigated is predominantly $d_{x^{2}-y^{2}}$ pairing, with no discernable presence of any time-reversal symmetry-breaking component within our experimental resolution. The spectra are analyzed using the generalized BTK theory, and the maximum of the $d$-wave superconducting gap $\Delta_{d}$ is found to scale with $T_{\infty}$, while the ratio of $\left(2 \Delta_{d} k_{B} T_{c}\right)$ increases with decreasing doping. These results are compared with the c-axis tunneling spectra of $\mathrm{Bi}_{2} \mathrm{Sr}_{2} \mathrm{CaCu}_{2} \mathrm{O}_{8+x}$, where the measured gap $\Delta^{*}$ appears to increase with decreasing doping, and the ratio of $\left(2 \Delta^{*} k_{B} T^{*}\right)$ is nearly independent of doping. On the other hand, the satellite features found in the quasiparticle spectra of both $\mathrm{YBa}_{2} \mathrm{Cu}_{3} \mathrm{O}_{7-5}$ and $\mathrm{Bi}_{2} \mathrm{Sr}_{2} \mathrm{CaCu}_{2} \mathrm{O}_{8+\mathrm{x}}$ appear to scale with their corresponding gaps. We have also conducted preliminary studies of spatially resolved quasiparticle spectra on a $(\mathrm{Zn}, \mathrm{Mg})$-substituted $\mathrm{YBa}_{2} \mathrm{Cu}_{3} \mathrm{O}_{7-\delta}$ single crystal, and have found two types of positively charged scattering centers, one at $\Omega_{A} \sim+2 \mathrm{meV}$ and the other at $\Omega_{B} \sim+10 \mathrm{meV}$. Finally, empirical revelation of nonequilibrium quasiparticle distributions in $\mathrm{YBa}_{2} \mathrm{Cu}_{3} \mathrm{O}_{7-8}$ under spin injection has been demonstrated for the first time in perovskite F-I-S heterostructures. The magnitude and temperature dependence of the spin diffusion length $\delta_{s}(T)$ are determined. It is found that $\delta_{s}(T)$ diverges more rapidly than that in conventional superconductors near $T_{c}$. More in-depth investigations are being conducted to elucidate the significance of $\delta_{5}(T)$ to the quasiparticle excitations in the spin and charge channels of cuprate superconductors.

\section{ACKNOWLEDGEMENT}

The work performed at Caltech is jointly supported by NSF Grant \#DMR-9705171 and NASA/OSS. Part of the research was performed by the Center for Space Microelectronics Technology, Jet Propulsion Laboratory, Caltech, through an agreement with the National Aeronautics and Space Administration. The work performed at SRL/ISTEC in Japan has been partially supported by NEDO.

\section{REFERENCES}

1. "Pairing symmetry and flux quantization in a tricrystal superconducting ring of $\mathrm{YBa}_{2} \mathrm{Cu}_{3} \mathrm{O}_{7}$ ", C. C. Tsuei, J. Kirtley, C. C. Chi et al., Phys. Rev. Lett. 73, 593 (1994).

2. "Phase sensitive tests of the symmetry of the pairing state in the high-temperature superconductors-- evidence for $d_{x^{2}-y^{2}}$ symmetry", D. J. van Harlingen, Rev. Mod. Phys. 67, 515 (1995); and references therein.

3. "Directional tunneling and Andreev reflection on $\mathrm{YBa}_{2} \mathrm{Cu}_{3} \mathrm{O}_{7}$ single crystals: Predominance of $d$-wave pairing symmetry verified with the generalized BTK theory", J. Y. T. Wei, N.-C. Yeh, D. F. Garrigus, and M. Strasik, Phys. Rev. Lett. 81, 2542 (1998).

4. "Observation of zero-bias tunneling conductance peak in underdoped $\mathrm{La}_{2-x} \mathrm{~S} \mathrm{r}_{x} \mathrm{Cu} \mathrm{O}_{4-\delta}$ ", J. Y. T. Wei, N.-C. Yeh, W. D. Si, and X. X. Xi, to appear in Physica B (1999).

5. "Directional tunneling spectroscopy studies of the temperature and doping dependence of the pairing symmetry in cuprate superconductors", N.-C. Yeh, J. Y. T. Wei, C.-T. Chen, W.-D. Si and X.-X. Xi, to appear in Physica C (2000).

6. "Temperature dependence of the half-integer magnetic flux quantum", J. R. Kirtley, C. C. Tsuei, and K. A. Moler, Science 285, 1373 (1999).

7. "Observation of bound surface states in grain-boundary junctions of high-temperature superconductors", L. Alff, A. Beck, R. Gross, A. Marx, S. Kleefisch, T. Bauch, H. Sato, M. Naito, and G. Koran, Phys. Rev. B. 58, 11197 (1998); "Spatially continuous zero-bias conductance peak on (110) $\mathrm{YBa}_{2} \mathrm{Cu}_{3} \mathrm{O}_{7-\delta}$ surfaces", L. Alff, H. Takashima, S. Kashiwaya, N. Terada, H. Thara, Y. Tanaka, M. Koyanagi, and K. Kajimura, Phys. Rev. B. 55, 14757 (1997).

8. "Tunneling spectroscopy of surface states of YBCO and NCCO", S. Kashiwaya et al., to appear in Physica C (2000).

9. "Midgap surface-states as a novel signature for $d_{x^{2}-y^{2}}$ wave superconductors", C. R. Hu, Phys. Rev. Lett. 72, 1526 (1994). 
10. "Theory of tunneling spectroscopy of d-wave superconductors", Y. Tanaka and S. Kashiwaya, Phys. Rev. Lett. 74, 3451 (1995).

11. "Observation of surface-induced broken time-reversal symmetry in $\mathrm{YBa}_{2} \mathrm{Cu}_{3} \mathrm{O}_{7}$ tunnel junctions", $\mathrm{M}$. Covington, $\mathrm{M}$. Aprili, E. Paraoanu, L. H. Greene, F. Xu, J. Zhu, and C. A. Mirkin, Phys. Rev. Lett. 79, 277 (1997).

12. "Tunneling into current-carrying surface states of high-Tc superconductors", M. Fogelstrom, D. Rainer, and J. A. Sauls, Phys. Rev. Lett. 79, 281 (1997).

13. " $\mathrm{C}$-cxis Josephson tunneling between $\mathrm{YBa}_{2} \mathrm{Cu}_{3} \mathrm{O}_{7-\delta}$ and $\mathrm{Pb}$ : Direct evidence for mixed order parameter symmetry in $\mathrm{a}$ high-temperature superconductor", K. A. Kouznetsov, A. G. Sun, B. Chen, A. S. Katz, S. R. Bahcall, J. Clarke, R. C. Dynes et al., Phys. Rev. Lett. 79, 3050 (1997).

14. "Coherence and single-particle excitations in the high-temperature superconductors", G. Deutscher, Nature 397, 410 (1999).

15. "Field induced and spontaneous sub-gap in [110] and [100] oriented YBCO films: indication for $a d_{x^{2}-y^{2}}+i d_{x y}$ order parameter", G. Deutscher, Y. Dagan, A. Kohen, and R. Krupke, to appear in Physica C (2000).

16. "Thermodynamics of superconducting lattice fermions", E. Otnes and A. Sudbø, Int. J. Mod. Phys. B 13, 1579 (1999).

17. "Local electronic structure of defects in superconductors", M. E. Flatte and J. M. Byers, Phys. Rev. B 80, 4546 (1998);

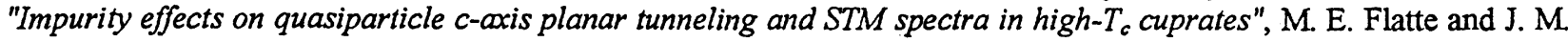
Byers, Phys. Rev. Lett. 56, 11213 (1997).

18. "Theory of scanning tunneling microscopy probe of impurity states in a d-wave superconductor", M. I. Salkola, A. V. Balatsky, and D. J. Scalapino, Phys. Rev. Lett. 77, 1841(1996); "Impurity-induced virtual bound state in d-wave superconductors", A. V. Balatsky, M. I. Salkola, and A. Rosengren, Phys. Rev. B 51, 15547 (1995);

19. "Competing orders and quantum criticality in doped antiferromagnmets", M. Vojta, Y. Zhang, and S. Sachdev, preprint, cond-mat/0003163 (2000).

20. "Understanding high-temperature superconductors: progress and prospects", D. Pines, Physica C 282-287, 273 (1997); "Nearly antiferromagnetic Fermi liquids: a progress report", Z. Phys. B 103, 129 (1997)

21. "Pairing in doped spin liquid - anyon versus d-wave superconductivity", D. S. Rokhsar, Phys. Rev. Lett. 70,493 (1993).

22. "Magnetic induction of $d_{x^{2}-y^{2}}+i d_{x y}$ order in high-temperature superconductors", R. B. Laughlin, Phys. Rev. Lett. 80, 5188 (1998); Physica (Amsterdam) 234C, 280 (1994).

23. M. Sigrist, D. B. Bailey, and R. B. Laughlin, Phys. Rev. Lett. 74, 3249 (1995).

24. For a comprehensive review of the experimental data related to the pseudogap, see "The pseudogap in high-temperature superconductors: an experimental survey", T. Timusk and B. Statt, Rep. Prog. Phys. 62, 61 (1999); and references therein.

25. See, for example, "Non-Fermi-liquid states and pairing instability of a general model of copper oxide metals", C. M. Varma, Phys. Rev. B 55, 14554 (1997); and references therein.

26. "Spectroscopic evidence for a pseudogap in the normal state of underdoped high-Tc superconductors", H. Ding, T. Yokaya, J. C. Campuzano, T. Takahashi, M. Randeria, M. N. Norman, T. Mochiku, K. Kadowaki, and J. Giapinzakis, Nature 382, 51 (1996).

27. "The resonating valence bond state in $\mathrm{La}_{2} \mathrm{CuO}_{4}$ and superconductivity", $\mathrm{P}$. W. Anderson, Science 235, 1196 (1987).

28. "Gauge-theory of the normal state of high-Tc superconductors", P. A. Lee and N. Nagaosa, Phys. Rev. B 46, 5621 (1992); "Ginzburg-Landu theory of the spin-charge-separated system", N. Nagaosa and P. A. Lee, Phys. Rev. B 45, 966 (1992).

29. "Unusual superconducting state of underdoped cuprates", P. A. Lee and X. G. Wen, Phys. Rev. Lett. 78, 4111 (1997).

30. "Importance of phase fluctuations in superconductors with small superfluid density", V. J. Emery and S. A. Kivelson, Nature 374, 434 (1995).

31. "Spin-gap proximity effect mechanism of high-temperature superconductivity", V. J. Emery, S. A. Kivelson, and O. Zachar, Phys. Rev. B 56, 6120 (1997).

32. "Topological phase-fluctuations, amplitude fluctuations, and criticality in extreme type-II superconductors", A. K Nguyen and A. Sudbo, Phys. Rev. B 60, (1999). 
33. "Vanishing of phase coherence in underdoped $\mathrm{Bi}_{2} \mathrm{Sr}_{2} \mathrm{CaCu}_{2} \mathrm{O}_{8+\delta}$ ", J. Corson, R. Mallozzi, J. Orenstein, J. N. Eckstein, Nature 398, 221 (1999).

34. "Nonequilibrium superconductivity under spin-polarized quasiparticle currents in perovskite ferromagnet-insulatorsuperconductor (F-I-S) heterostructures", N. -C. Yeh, R. P. Vasquez, C.-C. Fu, A. V. Samoilov, Y. Li, and K. Vakili, Phys. Rev. B 60, 10522 (1999).

35. "Tunneling spectroscopy study of spin-polarized quasiparticle injection effects in cuprate/manganite heterostructures", J. Y. T. Wei, N.-C. Yeh, C. C. Fu, and R. P. Vasquez, J. Appl. Phys. 85, 5350 (1999).

36. "Dynamic pair breaking in cuprate superconductors via injection of spin-polarized quasiparticles in perovskite F-I-S heterostructures", N.-C. Yeh, J. Y. T. Wei, C. C. Fu, and R. P. Vasquez, to appear in Physica B (1999).

37. "Critical current suppression in a superconductor by injection of spin-polarized carriers from a ferromagnet", V. A. Vas'ko, P. A. Kraus, K. R. Nikolaev, D. E. Grupp, C. A. Nordman, and A. M. Goldman, Phys. Rev. Lett. 78, 1134 (1997).

38. "Spin-polarized quasiparticle injection devices using $\mathrm{Au} / \mathrm{XBa}_{2} \mathrm{Cu}_{3} \mathrm{O}_{7} / \mathrm{LaAlO}_{3} / \mathrm{Nd}_{0.7 \mathrm{~S}} \mathrm{Sr}_{0.3} \mathrm{MnO}_{3}$ ", $\mathrm{Z}$. W. Dong, R. Ramesh, T. Venkatesan, M. Johnson, Z. Y. Chen, S. P. Pai, V. Talyansky, R. P. Sharma, R. Sheekala, C. J. Lobb, and R. L. Greene, Appl. Phys. Lett. 71, 1718 (1997).

39. "Spin conductivity and spin-charge seperation in the high- $T_{c}$ cuprates", Q. Si, Phys. Rev. Lett. 81, 3191 (1998).

40. "Spin injection into a Luttinger liquid", Q. Si, Phys. Rev. Lett. 78, 1767 (1997).

41. "Andreev reflection and spin injection into s- and d-wave superconductors", R. L. Merrill and Q. Si, Phys. Rev. Lett. 83, 5326 (1999).

42. "Nonaqueous chemical etch for $\mathrm{YBa}_{2} \mathrm{Cu}_{3} \mathrm{O}_{7-x}$ ", R. P. Vasquez, B. D. Hunt, and M. C. Foote, Appl. Phys. Lett. 53, 2692 (1988); "Reduced reactivities to air on $\mathrm{HF}$-treated $\mathrm{YBa}_{2} \mathrm{Cu}_{3} \mathrm{O}_{7-x}$ surfaces", R. P. Vasquez, B. D. Hunt, and M. C. Foote, Appl. Phys. Lett. 54, 2373 (1989).

43. "Wet chemical technique for passivation of $\mathrm{YBa}_{2} \mathrm{Cu}_{3} \mathrm{O}_{7-x}$ ", R. P. Vasquez, M. C. Foote, and B. D. Hunt, Appl. Phys. Lett. 55, 1801 (1989).

44. "Transition from metallic to tunneling regimes in superconducting micro-constrictions - excess current, charge imbalance, and super-current conversion", G. E. Blonder, M. Tinkham, and T. M. Klapwijk, Phys. Rev. B 25, 4515 (1982).

45. "Excitation gap in the normal state of $\mathrm{Bi}_{2} \mathrm{Sr}_{2} \mathrm{CaCu}_{2} \mathrm{O}_{8+\delta}$ ", A. G. Loeser, Z.-X. Shen, D. S. Dessau, D. S. Marshall, C. H. Park, P. Fourneir, and A. Kapitulnik, Science 273, 325 (1996); "Temperature and doping dependence of the Bi-Sr-Ca$\mathrm{Cu}-\mathrm{O}$ electronic structure and fluctuation effects", A. G. Loeser, Z.-X. Shen, M. C. Schabel, C. Kim, M. Zhang, A. Kapitulnik, and P. Fourneir, Phys. Rev. B 56, 14185 (1997).

46. "Pseudogap precursor of the superconducting gap in under- and overdoped $\mathrm{Bi}_{2} \mathrm{Sr}_{2} \mathrm{CaCu}_{2} \mathrm{O}_{8+\delta}$ ", Ch. Renner, B. Revaz, J.-Y. Genoud, K. Kadowaki, and O. Fischer, Phys. Rev. Lett. 80, 149 (1998); "Oxygen doping and temperature dependence of the tunneling spectroscopy on $\mathrm{Bi}_{2} \mathrm{Sr}_{2} \mathrm{CaCu}_{2} \mathrm{O}_{8+\delta}$ ", Ch. Renner, B. Revaz, J.-Y. Genoud, and O. Fischer, $J$. Low Temp. Phys. 105, 1083 (1996).

47. "Predominantly superconducting origin of large energy gaps in underdoped $\mathrm{Bi}_{2} \mathrm{Sr}_{2} \mathrm{CaCu} \mathrm{O}_{8+\delta}$ from tunneling spectroscopy", N. Miyakawa et al., Phys. Rev. Lett. 83, 1018 (1999); "Superconducting gap and pseudogap from tunneling conductance of $\mathrm{Bi}_{2} \mathrm{Sr}_{2} \mathrm{CaCu}_{2} \mathrm{O}_{8+\delta}$ with various oxygen concentration", N. Miyakawa et al., to appear in Physica $C(2000)$.

48. "Electronic spectra and their relation to the $(\pi, \pi)$ collective mode in high-T $T_{c}$ superconductors", J. C. Campuzano et al., Phys. Rev. Lett. 83, 3709 (1999); "Unusual dispersion and line shape of the superconducting state spectra of $\mathrm{Bi}_{2} \mathrm{Sr}_{2} \mathrm{CaCu}_{2} \mathrm{O}_{8+\delta}$ ", M. R. Norman, H. Ding, J. C. Campuzano, T. Takeuchi, M. Randeria, T. Yokaya, T. Takahashi, T. Mochiku, and K. Kadowaki, Phys. Rev. Lett. 79, 3506 (1997).

49. "Contribution to the theory of superconducting alloys with paramagnetic impurities", A. A. Abrikosov and L. P. Gor'kov, Soviet Phys. JETP 12, 1243 (1961).

50. "Superconductivity in strong spin-exchange field", P. Fulde and R. A. Ferrell, Phys. Rev. 135, A550 (1964).

51. "Effect of magnetic impurities on the density of states of superconductors", M. A. Wolf and F. Reif, Phys. Rev. 137, A557 (1965). 
52. "Zn and Ni doping effects on $T_{c}$ and spin gap behovior in $\mathrm{YBa}_{2} \mathrm{Cu}_{4} \mathrm{O}_{8}$ and $\mathrm{YBa}_{2} \mathrm{Cu}_{3} \mathrm{O}_{6.6}$ ", G.-q. Zheng, T. Odaguchi, Y. Kitaoka, K. Asayama, Y. Kodama, K. Mizuhashi, and S. Uchida, Physica C 263, 367 (1996); "Cu63 NQR NMR-study of Zn-substituted $\mathrm{YBa}_{2} \mathrm{Cu}_{4} \mathrm{O}_{8}$-- Effect of impurity on spin-gap-like behavior", G.-q. Zheng, T. Odaguchi, T. Mito, Y. Kitaoka, K. Asayama, and Y. Kodama, J. Phys. Soc. Japan 62, 2591 (1989).

53. "Correlations between magnetic and superconducting properties of $\mathrm{Zn}$-substituted $\mathrm{YBa}_{2} \mathrm{Cu}_{3} \mathrm{O}_{6+x}$ ", $\mathrm{H}$. Alloul, P. Mendels, H. Casalta, J. F. Marucco, and J. Arabski, Phys. Rev. Lett. 67, 3140 (1991).

54. "Superconducting and normal-state properties of $\mathrm{YBa}_{2}\left(\mathrm{Cu}_{1-x} \mathrm{Zn}_{x}\right)_{4} \mathrm{O}_{8}$ ", T. Miyatake, K. Yamaguchi, T. Takata, N. Koshizuka, S. Tanaka, Phys. Rev. B 44, 10139 (1991).

55. "Zn-substitution effects on the optical conductivity $\mathrm{YBa}_{2} \mathrm{Cu}_{3} \mathrm{O}_{7-\delta}$ crystals: Strong pair breaking and reduction of in-plane anisotropy", N. L. Wang, S. Tajima, A. I. Rykov, and K. Tomimoto, Phys. Rev. B 57, R11081 (1999).

56. "Impurity effects on the superconducting coherence length in $\mathrm{Zn}$ - or Ni-doped $\mathrm{YBa}_{2} \mathrm{Cu}_{3} \mathrm{O}_{6.9}$ single crystals ", $\mathrm{K}$. Tomimoto, I. Terasaki, A. I. Rykov, T. Mimura, and S. Tajima, Phys. Rev. B 60, (1999).

57. "Imaging the effects of individual zinc impurity atoms on superconductivity in $\mathrm{Bi}_{2} \mathrm{Sr}_{2} \mathrm{CaCu}_{2} \mathrm{O}_{8+\delta}$ ", S. H. Pan, E. W. Hudson, K. M. Lang, H. Eisaki, S. Uchida, and J. C. Davis, Nature 403, 746 (2000).

58. "STM study of effects on superconductivity of individual zinc and nickel impurity atoms in $\mathrm{Bi}_{2} \mathrm{Sr}_{2} \mathrm{CaCu}_{2} \mathrm{O}_{8+\delta}$ ", J. C. Davis, S. H. Pan et al., to appear in Physica C (2000).

59. For Friedel oscillation and sum rule related to the theory of alloys, see J. Friedel, Phil. Mag. Supplement 3, 446 (1954); Nuovo cimento supplemento 7, 287 (1958).

60. For review articles, see K. E. Gray (ed.), "Nonequilibrium Superconductivity, Phonons, and Kapitza Boundaries", NATO ASI Series, Plenum, New York (1981).

61. "Spin-polarized quasiparticle transport in ferromagnet-d-wave superconductor junctions", J. X. Zhu, B. Friedman, and C. S. Ting, Phys. Rev. B 59, 9558 (1999).

62. "Spin current in ferromagnet-insulator-superconductor junctions", S. Kashiwaya, Y. Tanaka, N. Yoshida, and M. R. Beasley, Phys. Rev. B 60, 3572 (1999).

63. "Tunneling spectroscopy for ferromagnet/superconductor junctions", I. Zutic and O. T. Valls, Phys. Rev. B 61, 1555 (2000).

64. "Spin-dependent tunneling into ferromagnetic nickel", P. M. Tedrow and R. Meservey, Phys. Rev. Lett. 26, 192 (1971); "Spin polarization of electrons tunneling from films of $\mathrm{Fe}, \mathrm{Co}, \mathrm{Ni}$, and Gd", Phys. Rev. B 7, 318 (1973); "Correlation between spin polarization of tunnel currents from $3 d$ ferromagnets and their magnetic moments", R. Meservey, D. Paraskevopoulos, and P. M. Tedrow, Phys. Rev. Lett. 37, 858 (1976).

65. "Interfacial charge-spin coupling -- injection and detection of spin magnetization in metals", M. Johnson and R. H. Silsbee, Phys. Rev. Lett. 55, 1790 (1985); "Thermodynamic analysis of interfacial transport and of the thermomagnetoelectric system", M. Johnson and R. H. Silsbee, Phys. Rev. B 35, 4959 (1987).

66. "Spin-polarized transport", G. A. Prinz, Physics Today 48, No.4, 58 (1995); and references therein.

67. M. I. Salkola, A. V. Balatsky, and J. R. Schrieffer, Phys. Rev. B 55, 12648 (1997).

68. "Paramagnetic state in d-wave superconductors", H. Won, H. Jang, and K. Maki, preprint, cond-mat/9901252; "Pauli paramagnetism and impurity effects in d-wave superconductors", $\mathrm{K}$ Maki, Y. Seo, and $\mathrm{H}$. Won, to appear in Physica B, (2000). 